\title{
Smart Vehicle Parking Management using IOT
}

\author{
Gayathri Chayanam ${ }^{1}$, Dr. Kalavathi ${ }^{2}$ Sravya $^{3}$ \\ 1(Information Technology, VasireddyVenkatadri Institute of Technology, Guntur) \\ 2 (Professor, Information Technology, VasireddyVenkatadri Institute of Technology, Guntur) \\ 3 (Information Technology, VasireddyVenkatadri Institute of Technology, Guntur)
}

\section{Abstract:}

Smart city is another powerful application of IOT generating curiosity among world's population. Smart surveillance, automated transportation, smarter energy management systems, water distribution, urban security and environmental monitoring all are examples of internet of things applications for smart cities. It's inevitable for the people to update with the growing technology. And generally people are facing problems on parking vehicles in parking slots in a city. In this study we design a Smart Vehicle Parking Management System (SVPMS) which enables the user to find the nearest parking area and gives availability of parking slots in that respective parking area and allows the user to make a reservation for their vehicle in the parking slots. And it mainly focuses on reducing the time in finding the parking lots. Thus it reduces the fuel consumption which in turn reduces carbon footprints in an atmosphere.

Keywords - IOT, sensors, mobile app, Wi-Fi.

\section{INTRODUCTION}

The purpose for developing this type of software or introducing this Smart Parking System is to facilitateeveryone who is concerned to get a parking slot at their destination places. This is achieved by using the IOT technology and provides a better automated solution than earlier. The hunger for automation brought many revolutions in the existing technologies. These technologies can be used in our day to day life which increases the growth of technology and automation and reduces the chance of accidents happening. Considering the advantages of $\mathrm{Wi}-\mathrm{Fi}$ an advanced automation system was developed to monitor the status of parking slots and make a reservation for the vehicle in their desired parking area and slot if available. Wi-Fi (Short for Wireless Fidelity) is a wireless technology that uses radio frequency to transmit data through the air. This system uses Wi-Fi to provide reservation and to maintain them in the server. The controlling device for the monitoring in the project is a Microcontroller (Aurdinonano). The data is read by Microcontroller which sends the data over Wi-Fi to the IOT mobile app. The Microcontroller is programmed used embedded „C, language.
An embedded system is a combination of software and hardware to perform a dedicated task. Some of the main devices used in embedded products are Microprocessors and Microcontrollers. Microprocessors are commonly referred to as general purpose processors as they simply accept the inputs, process it and give the output. In contrast, a microcontroller not only accepts the data as inputs but also manipulates it, interfaces the data with various devices, controls the data and thus finally gives the result. In this project instead of providing parking information to a driver and letting him make a parking decision, the "smart parking" system assigns and reserves an optimal parking space based on the driver's requirements that combine proximity to destination and parking cost, while also ensuring that the overall parking capacity is efficiently utilized.The project "IOT based Smart Vehicle Parking Management System" was designed such that the status of parking slots and the reservation for the vehicles can be known from anywhere in the users mobile app. 


\section{Procedural details}

In this section the architecture of the parking management system and design aspect of independent modules are stated.

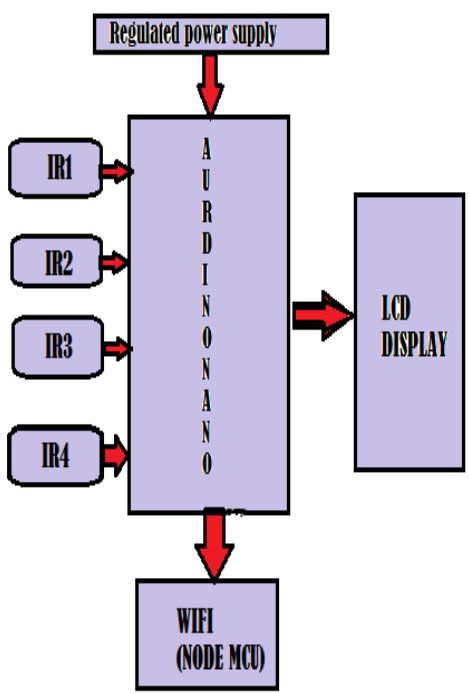

Fig1: Architecture

The main components involved in this work are:

1. Micro controller(ATmega328/P)

2. Aurdinonano

3. Regulated power supply (RPS)

4. LED Indicator

5. Wi-Fi module (ESP8266)

6. IR sensor

7. LCD display

\section{A) MICROCONTROLLER}

The Atmel ${ }^{\circledR}$ picoPower ${ }^{\circledR}$ ATmega328/P is a lowpower CMOS 8-bit microcontroller based on the $\mathrm{AVR}^{\circledR}$ enhanced RISC architecture. By executing powerful instructions in a single clock cycle, the ATmega328/P achieves throughputs close to 1MIPS per MHz. This empowers system design to optimize the device for power consumption versus processing speed. The Atmel $\mathrm{AVR}^{\circledR}$ core combines a rich instruction set with 32 general purpose working registers. All the 32 registers are directly connected to the Arithmetic Logic Unit (ALU), allowing two independent registers to be accessed in a single instruction executed in one clock cycle. The resulting architecture is more code efficient while achieving throughputs up to ten times faster than conventional CISC microcontrollers.

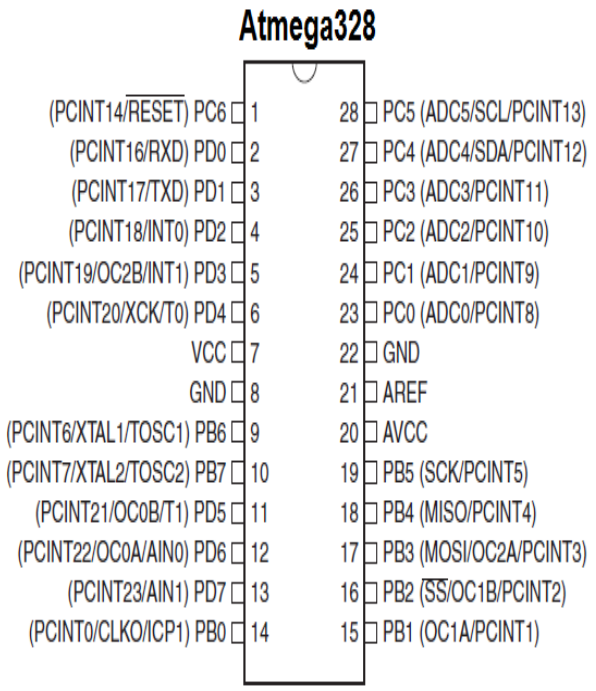

Fig 2: Pin Configuration of the Micro Controller

\section{B) AURDINO}

The Aurdino Nano is a small, complete and breadboard-friendly board based on the Atmega 328 (Airdino Nao3.0 or ATmega 168 (Arduino Nano 2.x). It has more or less the same functionality of the ArduinoDuemilanove, but in a different package. It lacks only a DC powe jack, and works with a Mini-B USB cable instead of a standard one. The Nano was designed and is being produced by Gravitech.

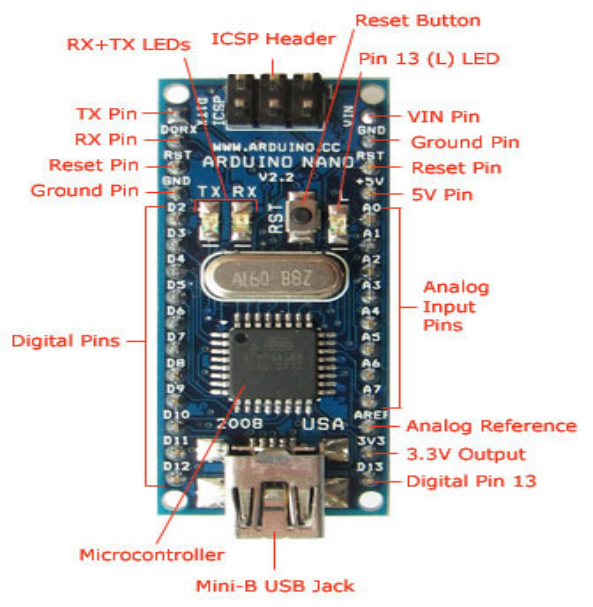

Fig 3. Pin description of aurdinonano 


\section{C) REGULATD POWE SUPPLY}

In this work, power supplies with $+5 \mathrm{~V} \&-5 \mathrm{~V}$ option normally $+5 \mathrm{~V}$ is enough for total circuit. Another (-5V) supply is used in case of OP amp circuit .Transformer primary side has $230 / 50 \mathrm{HZ}$ AC voltage whereas at the secondary winding the voltage is step downed to $12 / 50 \mathrm{hz}$ and this voltage is rectified using two full wave rectifiers .the rectified output is given to a filter circuit to fitter the unwanted ac in the signal After that the output is again applied to a regulator LM7805(to provide $+5 \mathrm{v}$ ) regulator. Whereas LM7905 is for providing $-5 \mathrm{~V}$ regulation. $(+12 \mathrm{~V}$ circuit is used for stepper motors, Fan and Relay by using LM7812 regulator same process like above supplies.)

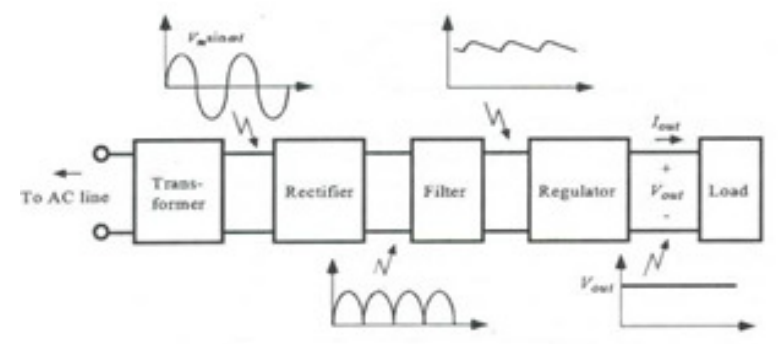

Fig 4.Components of regulated power supply

The components mainly used in above figure are:-

- Transformer

- Rectifier

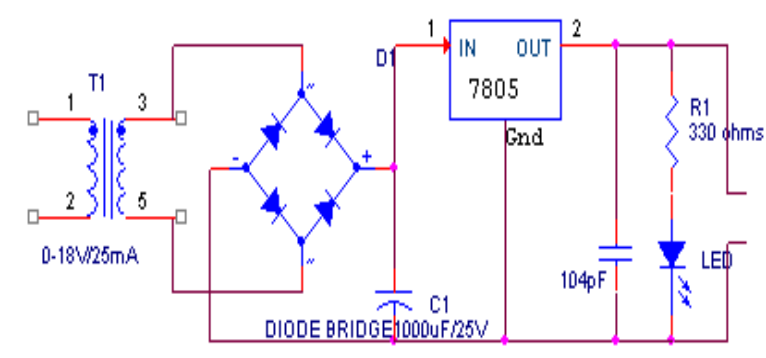

Fig 5. Regulated Power Supply Circuit

- Filter

- Voltage regulator

D) LED INDICATOR
A light-emitting diode (LED) is a two-lead semi conductor light source. It is a p-n junction diode that emits light when activated. When a suitable current is applied to the leads, electrons are able to recombine with electron holes within the device, releasing energy in the form of photons. This effect is called electroluminescence, and the color of the light (corresponding to the energy of the photon) is determined by the energy band gap of the semiconductor. LEDs are typically small (less than $1 \mathrm{~mm}^{2}$ ) and integrated optical components may be used to shape the radiation pattern.

Appearing as practical electronic components in 1962, the earliest LEDs emitted low-intensity infrared light. Infrared LEDs are still frequently used as transmitting elements in remote-control circuits, such as those in remote controls for a wide variety of consumer electronics. The first visible-

light LEDs were of low intensity and limited to red. Modern LEDs are available across the visible, ultraviolet, and infrared wavelengths, with very high brightness.

Fig 6. Led Indicator

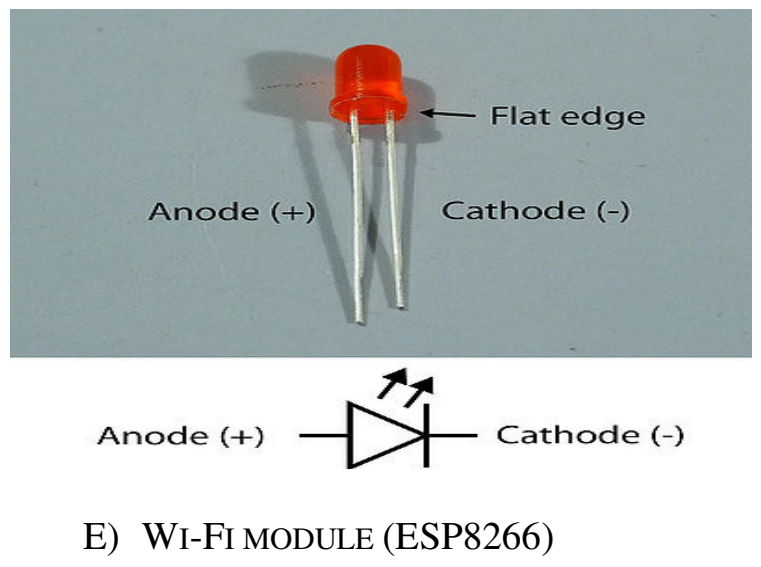

The NodeMcu is an open-source firmware and development kit that helps you to Prototype your IOT product within a few Lua script lines. Features:

$\checkmark$ Open-source

$\checkmark$ Interactive 
$\begin{array}{cl}\checkmark & \text { Programmable } \\ \checkmark & \text { Low cost } \\ \checkmark & \text { Simple } \\ \checkmark & \text { Smart } \\ \checkmark & \text { WI-FI enabled }\end{array}$

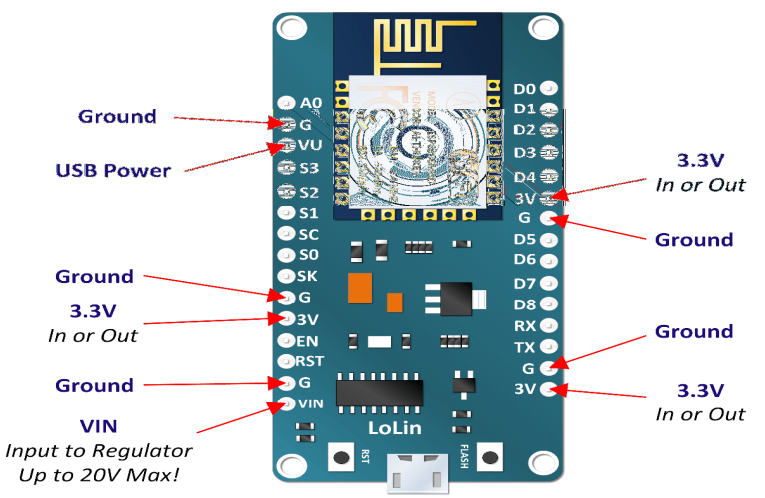

Fig 7. WI-FI adapter

\section{F) IR SENSOR}

Infrared (IR) light is electromagnetic radiation with longer wavelengths than those of visible light, extending from the nominal red edge of the visible spectrum at 700 nanometers $(\mathrm{nm})$ to $1 \mathrm{~mm}$. This range of wavelengths corresponds to a frequency range of approximately $430 \mathrm{THz}$ down to $300 \mathrm{GHz}$. Most of the thermal radiation emitted by objects near room temperature is infrared.

Infrared light is used in industrial, scientific, and medical applications. Night-vision devices using active near-infrared illumination allow people or animals to be observed without the observer being detected. Infrared astronomy uses sensorequipped telescopes to penetrate dusty regions of space, such as molecular clouds; detect objects such as planets, and to view highly redshifted objects from the early days of the universe. Infrared thermal-imaging cameras are used to detect heat loss in insulated systems, to observe changing blood flow in the skin, and to detect overheating of electrical apparatus.

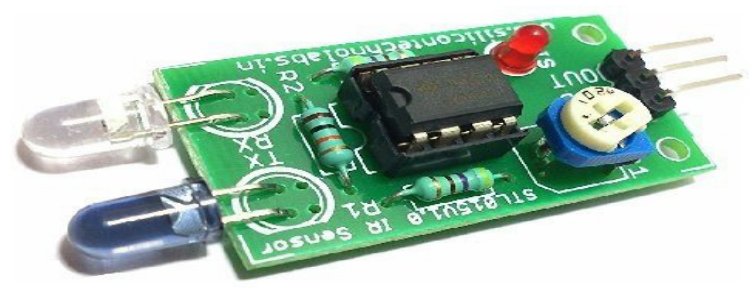

Fig 8.IR Sensor

\section{EXPERIMENTAL RESUlTS}

The "IOT based smart vehicle parking Management System" was designed such that the status of parking slots and their booking can be known and done from anywhere in the users app. This is achieved using Wi-Fi communication. In this system, the user has to be connected to the Wi-Fi network in the mobile through which he is given access to the app and can know about the status of their reserved parking slot or their availability.
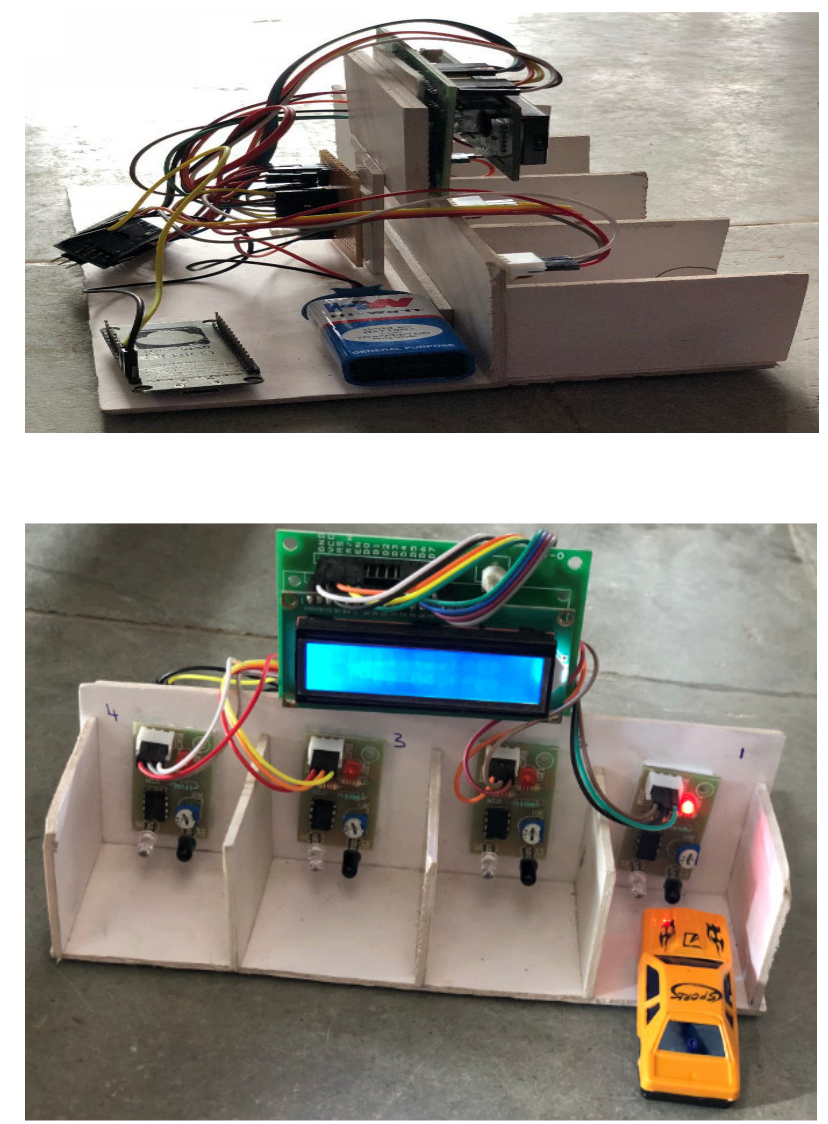

Fig 9: Smart Parking System

V. CONCLUSION 
The objectives of this project have been achieved. The hassle in searching for available parking lots has been completely eliminated by reserving the lots via IOT system. The security feature of the system is enhanced with the email verification upon entrance to the parking lot.The designed system could be applied everywhere due to its ease of usage and effectiveness.

\section{FUTURE SCOPE}

This work can be extended by adding an application of online verification of the security through number plate scanner or through QR code scanner.

\section{REFERENCES}

1. L. Atzori, A. Iera, and G. Morabito, "The Internet of things: a survey," Computer Networks, vol. 54, no. 15.

2. Bilodeau, V.P. Intelligent Parking Technology Adoption. Ph.D. Thesis, University of Southern Queensland: Queensland, Australia, 2010.

3. Li, T.S.; Ying-Chieh, Y.; Jyun-Da, W.; Ming-Ying, H.; Chih-Yang, C. Multifunctional intelligent autonomous parking controllers for carlike mobile robots. IEEE Trans. Ind. Electron. 2010, 57, 16871700 .

4. Faheem1, S.A. Mahmud, G.M. Khan, M. Rahman and H. Zafar,\|A Survey of Intelligent Car Parking System\|, October 2013

5. S. Alam, M. M. R. Chowdhury, and J. Noll, "Senaas: An event-driven sensor virtualization approach for internet of things cloud," in Networked Embedded Systems for Enterprise Applications (NESEA), 2010 IEEE International Conference on, November 2010, pp. 1-6. [Online]. Available:

http://dx.doi.org/10.1109/NESEA.2010.5678060

6. Choeychuen, K. Automatic parking lot mapping for available parking space detection. In Proceedings of the 5th International Conference on Knowledge and Smart Technology (KST), Chonburi, Thailand, 31 January-1 February 2013; pp. 117-121.
7. Z. Schelby, K. Hartke, and C. Bormann, (Aug. 28, 2013) "Constrained application protocol (CoAP)," CoRE Working Group Internet-Draft. [Online]. Available: http://datatracker.ietf.org/doc/draft-ietf-core-coap/

8. Keat, C.T.M.; Pradalier, C.; Laugier, C. Vehicle detection and car park mapping using laser scanner. In Proceedings of the IEEE/RSJ International Conference on Intelligent Robots and Systems, Edmonton, AB, Canada, 2-6 August 2005; pp. 2054-2060. 9

9. http://www.ijetsr.com/images/short_pdf/149502134 7 nitttr888 ijetsr.pdf

10. https://create.arduino.cc/projecthub/taifur/smartparking-system-b3f5a0

11. http://www.instructables.com/id/Smart-Parking/

12. http://www.mdpi.com/journal/sensorsSensors 2014, 14, 22372-22393; doi:10.3390/s141222372

13. http:ijarcet.org/wpcontent/uploads/IJ 